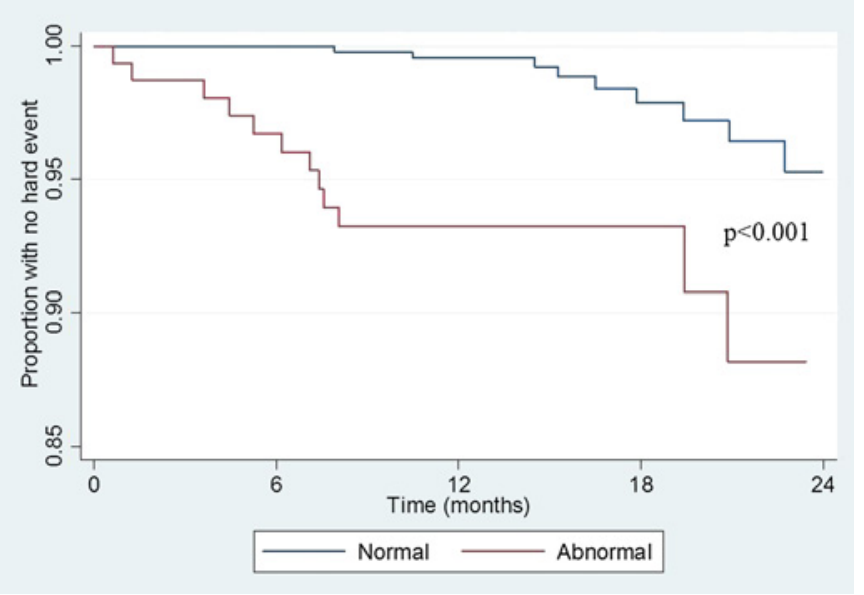

Abstract 101 Figure 2 Kaplan-Meier survival estimate of time to hard event.

\section{POCKET-SIZE HAND-HELD CARDIAC ULTRASOUND IN THE HANDS OF STUDENTS AND JUNIOR DOCTORS: DOES IT IMPROVE DIAGNOSTIC ACCURACY OVER HISTORY, PHYSICAL EXAMINATION AND ECG?}

doi:10.1136/heartjnl-2012-301877b.102

V F Panoulas, * A L Daigeler, A S Lota, A S N Malaweera, D Baskaran, S Rahman, P Nihoyannopoulos. Hammersmith Hospital, Imperial Healthcare NHS Trust, London, UK

Background Even though patient history taking and physical examination remain the foundations of patient evaluation in daily clinical practise, there has been a decline in the accuracy of the later. Pocket hand held echocardiographic (PHHE) devices have recently been introduced and could potentially improve diagnostic accuracy in the hands of non-cardiologists. The amount of training required to achieve optimal results remains a matter of debate.

Aim We hypothesised that use of PHHE after limited training, can improve diagnostic accuracy even in the hands of medical students and inexperienced physicians.

Methods Five final year medical students and 3 junior doctors without prior echocardiographic experience participated in a standardised 2-h PHHE bed-side training program. Subsequently they assessed 122 patients (history taking, physical examination, ECG interpretation and PHHE). Their physical and echocardiographic findings were compared to those of a transthoracic echocardiography accredited cardiologist.

Results A total of $122 \mathrm{~V}$-scans were performed of which 64 (53\%) by final-year medical students and $58(47 \%)$ by junior doctors. Mean age of the participants was $64 \pm 16.1$ years and $87(71.3 \%)$ were male. Out of 122 patients, 69 (56.6\%) had LV dysfunction, 16 (13.1\%) had RV dysfunction, 74 (52.5\%) had valvular abnormalities, 5 (4.1\%) had prosthetic valves, $6(4.9 \%)$ had pericardial effusions and 4 (3.3\%) ascending aorta disease. Mean $\pm S D$ for diagnostic accuracy (maximum=1) after history, physical examination and ECG interpretation was $0.53 \pm 0.19$ whereas addition of PHHE increased its value to $0.85 \pm 0.2(\mathrm{Z}=-8.964, \mathrm{p}<0.001)$. In $88(73.3 \%)$ patients there was concordance between cardiologist and trainees in LV assessment, in 23 $(19.1 \%)$ trainees underestimated or overestimated LV by one grade and in $9(7.4 \%)$ by at least two grades. When assessing for presence of moderate to severe left ventricular dysfunction by means of history and physical examination specificity was $84.9 \%$ and sensitivity only $25.9 \%$ whereas after PHHE these figures raised to $93.6 \%$ and $74.1 \%$ respectively. There were a total of 94 valvular lesions (present in 74 patients), 10 of which (10.6\%) were stenotic and 84 (89.3\%) regurgitant. There was a total of 40 moderate to severe valvular regurgitation lesions. Trainee sensitivity in identifying the afore mentioned lesions was $70 \%$ whereas specificity $98 \%$. Regarding moderate to severe valvular stenosis sensitivity was $85.7 \%$ whereas specificity was $100 \%$. Ausculatation for presence of valvular abnormality (without specifying which valve or what kind of abnormality) revealed a $93.8 \%$ specificity and a $45.9 \%$ sensitivity.

Conclusions In the current study use of PHHE after brief, bed-side training greatly improved the diagnostic accuracy of medical students and junior doctors, over and above history, physical examination and ECG interpretation.

\section{3 \\ AORTIC INFLAMMATION IS REDUCED, AND PARALELLES CHANGES IN AORTIC STIFFNESS BY ANTI-TNF $\alpha$ THERAPY IN RHEUMATOID ARTHRITIS}

doi:10.1136/heartjnl-2012-301877b.103

K M Maki-Petaja, ${ }^{*}$ M Elkhawad, F Joshi, F Hall, A Ostor, J Cheriyan, J H Rudd, I B Wilkinson. University of Cambridge, Cambridge, UK

Background Rheumatoid arthritis (RA) is a systemic inflammatory condition associated with increased cardiovascular risk which is not fully explained by traditional risk factors. Endothelial dysfunction and increased aortic stiffness may mediate some of the increased risk. Additionally, there may be direct vascular inflammation which could directly accelerate atherosclerosis. We hypothesised that patients with RA exhibit a subclinical aortic vasculitis which can be reversed with anti-tumour necrosis factor $\alpha$ (TNF) therapy.

Methods The aortas and carotid arteries of 15 patients with severe rheumatoid arthritis were imaged before and after anti-TNF therapy using 18F-fluoro-deoxyglucose positron emission tomography (FDG-PET) with CT co-registration. Tracer uptake was analysed in various arterial segments by measuring maximum standard uptake values (SUV) and subsequently corrected for blood uptake to obtain a target to background ratio (TBR). Carotid to femoral pulse wave velocity (PWV) as a measure of aortic stiffness, disease activity and inflammatory biomarkers were also measured.

Results Mean baseline aortic TBR was 2.07 \pm 0.20 . Following antiTNF $\alpha$ therapy, there was a significant reduction in abdominal aortic TBR $(-0.18 \pm 0.27, p=0.03)$ and in the most diseased segment in the whole aorta $(-0.48 \pm 0.59, \mathrm{p}=0.01)$. TBR was also reduced in all other aortic segments and the proportion of hot slices (defined as TBR $>1.9$ ) was reduced by $31 \%$, but these did not reach statistical significance. There was no change in carotid TBR following treatment. Aortic PVW was reduced by $0.43 \pm 1.0 \mathrm{~m} / \mathrm{s}, \mathrm{p}=0.1$ ) and there was a significant correlation between a reduction in aortic PWV and abdominal TBR $(\mathrm{R}=0.57, \mathrm{p}=0.03)$ and between aortic PWV and proportion of "hot" slices $(R=0.66, p=0.01)$. There was a concomitant reduction in serum CRP $(-8 \pm 12 \mathrm{mg} / \mathrm{l}, \mathrm{p}=0.02)$ and disease activity (DAS28 $-1.41 \pm 1.51, \mathrm{p}=0.002$ ).

Conclusions This study demonstrates for the first time that patients with RA have high aortic and carotid FDG uptake, suggesting subclinical vasculitis. Moreover, they exhibit a reduction in FDG uptake following anti-TNF $\alpha$ therapy, which correlated with a reduction in aortic stiffness. These results suggest that subclinical vasculitis could be the mechanism behind the increased cardiovascular risk and that effective treatment of inflammation may help to reduce the cardiovascular risk in this patient population.

\section{ENHANCING ENDOTHELIAL INSULIN SENSITIVITY REDUCES NITRIC OXIDE BIOAVAILABILITY: A ROLE FOR NADPH OXIDASE-DERIVED REACTIVE OXYGEN SPECIES}

doi:10.1136/heartjnl-2012-301877b.104

H Viswambharan, ${ }^{*}$ P Sukumar, M C Gage, N Yuldasheva, A Skromna, H Imrie, S Galloway, J Smith, R Cubbon, S B Wheatcroft, M T Kearney. University of Leeds, Leeds, UK

Introduction Insulin resistance at a whole body level and in the endothelium precedes type 2 diabetes. Insulin resistance at the level 
of the endothelium reduces bioavailability of the anti-atherosclerotic signalling radical nitric oxide (NO). We explored the effects of increasing insulin signalling in the endothelium, using novel transgenic mice, over-expressing Type A human Insulin Receptor (HIRECO) in the endothelium, driven by the Tie-2 promoterenhancer.

Methods Semi-quantitative RT-PCT was carried out on various tissues and isolated endothelial cells from lungs to confirm significant levels of human insulin receptor mRNA while the protein expression was confirmed by western blotting on aortic sections or endothelial cells. Lucigenin-enhanced chemiluminescence was exploited to measure superoxide anion levels while; vasomotor functions were assessed in thoracic aortic rings mounted in organ baths.

Results No significant changes in morphological features, metabolic phenotypes or blood pressure regulation were observed between the HIRECO and wild type (WT) littermates. However, plasma insulin levels were similar following an overnight fast, but were decreased in the HIRECO after glucose challenge. HIRECO mice demonstrated significant endothelial dysfunction measured by a blunted response to acetylcholine (Emax, WT vs HIRECO: $84 \pm 3 \%$ vs $68 \pm 3 \%$ respectively; $\mathrm{n}=5, \mathrm{p}>0.05)$. Endothelium-independent response to sodium nitroprusside remained unchanged. The impaired aortic response to acetylcholine was normalised by the specific NADPH oxidase inhibitor peptide, gp91ds-tat, (Emax: $93 \pm 5 \%$; $=6, p<0.05$ ), as well as the superoxide dismutase mimetic, $\mathrm{Mn}$ (III) tetrakis (1-methyl-4-pyrydil) porphyrin pentachloride. Isolated aortic rings of HIRECO exhibited a hypercontractile response to phenylephrine compared to wild type mice (log EC50, WT vs HIRECO: $6.96 \pm 0.03$ vs $7.24 \pm 0.08, \mathrm{n}=6$, $\mathrm{p}<0.01)$. Indeed, HIRECO mice elicited a 1.65-fold increase in the level of superoxide anion production compared to WT. Basal NO bioactivity was decreased in HIRECO compared to WT littermates (Emax upon exposure to eNOS inhibitor, L-NAME in phenylephrineconstricted aorta, WT vs HIRECO: $144 \pm 27.9 \%$ vs $32 \pm 33 \%, n=5$, $\mathrm{p}<0.05)$. However, basal eNOS phosphorylation levels in isolated endothelial cells of HIRECO mice was enhanced 1.56-fold compared to WT littermates.

Conclusions/Implications These data demonstrate enhanced oxidative stress in a novel murine model of increased insulin signalling in the endothelium, leading to reduced bioavailability of nitric oxide and atherosclerosis. These data also demonstrate for the first time, that increased insulin sensitivity in the endothelium, increases the generation of superoxide anion and reduces NO bioavailability.

Funding This work was supported by The British Heart Foundation.

\section{NOX2 NADPH-OXIDASE A NOVEL TARGET TO PREVENT INSULIN RESISTANCE RELATED ENDOTHELIAL CELL DYSFUNCTION}

doi:10.1136/heartjnl-2012-301877b.105

${ }^{1} \mathrm{P}$ Sukumar, ${ }^{* 1} \mathrm{H}$ Viswambharan, ${ }^{1} \mathrm{H}$ Imrie, ${ }^{1} \mathrm{R} \mathrm{M}$ Cubbon, ${ }^{1} \mathrm{~N}$ Yuldasheva, ${ }^{1} \mathrm{M}$ Gage, 'S Galloway, ${ }^{1} \mathrm{~A}$ Skromna, ${ }^{1} \mathrm{~J}$ Smith, ${ }^{1} \mathrm{~K}$ Gatenby, ${ }^{1} \mathrm{D}$ J Beech, ' $\mathrm{S}$ B Wheatcroft, ${ }^{2} \mathrm{~K}$ M Channon, ${ }^{3} \mathrm{~A}$ M Shah, ${ }^{1} \mathrm{M}$ T Kearney. ${ }^{1}$ University of Leeds, Leeds, UK; ${ }^{2}$ University of Oxford, Oxford, UK; ${ }^{3}$ Kings College, London, UK

Introduction Insulin resistance, a central pathophysiological feature of type 2 diabetes is characterised by a deleterious change in endothelial cell phenotype, a hallmark of which is increased generation of reactive oxygen species. We examined the role of NADPH oxidase and specifically NOX2 NADPH oxidase in insulin resistance induced endothelial cell dysfunction. We studied mice with endothelium specific over expression of a dominant negative insulin receptor (ESMIRO) and mice with whole body haploinsufficiency of the insulin receptor $\left(\mathrm{IR}^{+/-}\right)$.
Methods ESMIRO mice, a model of endothelium specific insulin resistance, and $\mathrm{IR}^{+/-}$mice a model of whole body insulin resistance were used to examine the effect of acute and chronic pharmacological inhibition of NADPH oxidase on superoxide production (lucigenin enhanced chemiluminescence) and endothelial function (acetylcholine mediated aortic relaxation). To specifically investigate the role of NOX2, we crossed mice with holoinsufficiency of NOX2 with ESMIRO mice to generate ESMIRO/NOX2 $2^{\mathrm{y}-}$ mice. Data expressed as mean \pm SEM; male mice used for all experiments.

Results Basal superoxide generation in isolated pulmonary endothelial cells (PEC) was increased in both models of insulin resistance (by $130 \%$ in ESMIRO and $220 \%$ in $\mathrm{IR}^{+/-}$compared to wild type, both $\mathrm{p}<0.01 ; \mathrm{n}=3$ for each group). Pre-treating PEC with gp91dstat, a cell permeable specific blocker of NOX subunit assembly and function, reduced the excessive superoxide generation in ESMIRO and $\mathrm{IR}^{+/-}$. Endothelial $\mathrm{NO}$ mediated vasorelaxation in aortic rings from ESMIRO and $\mathrm{IR}^{+/-}$was impaired $(101 \% \pm 11 \%$ relaxation to $1 \mu \mathrm{M}$ acetylcholine in wild type, $61 \% \pm 3 \%$ in ESMIRO $(n=5$, $\mathrm{p}<0.01) ; 91 \% \pm 3 \%$ relaxation in wild type, $75 \% \pm 6 \%$ in $\mathrm{IR}^{+/-}(\mathrm{n}=4$, $\mathrm{p}=0.03)$ ). This was restored by pre-incubating rings with gp91ds-tat ( $92 \% \pm 6 \%$ relaxation in ESMIRO and $93 \% \pm 6 \%$ in $\mathrm{IR}^{+/-}$). Chronic (4 weeks) administration of gp91-ds tat peptide (using osmotic mini-pump) to ESMIRO and $\mathrm{IR}^{+/-}$mice also restored endothelial dependent relaxation (from $83 \% \pm 11 \%$ to $100 \% \pm 9 \%$ in ESMIRO and to $136 \% \pm 11 \%$ in $\mathrm{IR}^{+/-}$). NOX2 gene expression was significantly higher in ESMIRO mice. ESMIRO/NOX2 ${ }^{\mathrm{y} /-}$ mice with complete deletion of NOX2 had significantly greater relaxation responses to acetylcholine than ESMIRO $(77 \% \pm 6 \%$ relaxation in ESMIRO and $100 \% \pm 4 \%$ in ESMIRO/NOX2 ${ }^{\mathrm{y} /-} ; \mathrm{n}=5, \mathrm{p}=0.002$ ). Neither pharmacological nor genetic inhibition of NADPH oxidase had any effect on glucose homeostasis.

Discussion These data in complementary models of insulin resistance demonstrate that acute or chronic pharmacological inhibition of NADPH oxidase reduces superoxide generation and improves endothelial function. Specifically targeting NOX2 also restored endothelial function in ESMIRO mice.

Funding This work was supported by the Medical Research Council UK.

\section{ROLE OF NEURONAL VS ENDOTHELIAL NITRIC OXIDE SYNTHASE IN THE CORONARY BLOOD FLOW RESPONSE TO PACING}

doi:10.1136/heartjnl-2012-301877b.106

${ }^{1} \mathrm{H}$ Shabeeh, ${ }^{* 1} \mathrm{~N}$ Melikian, ${ }^{1} \mathrm{R}$ Dworakowski, ${ }^{2} \mathrm{~B}$ Casadei, ${ }^{1} \mathrm{P}$ Chowienczyk, ${ }^{1} \mathrm{~A}$ M Shah. ${ }^{1}$ King's College London British Heart Foundation Centre of Research Excellence, London, UK; ${ }^{2}$ Department of Cardiovascular Medicine, University of Oxford, Oxford, UK

Background Endothelial nitric oxide synthase (eNOS) has been assumed to be the major source of nitric oxide (NO) regulating human coronary blood flow (CBF). In recent first-in-human studies with a neuronal NOS (nNOS)-selective inhibitor, we provided evidence that nNOS-derived NO regulates basal coronary blood flow whereas eNOS mediates increases in flow in response to the endothelial agonist, substance $\mathrm{P}$. The present study aimed to investigate the effects of nNOS vs eNOS inhibition on coronary blood flow response to increased heart rate.

Methods We studied the effects of the nNOS-selective inhibitor, S-methyl-L-thiocitrulline (SMTC), and the non-selective NOS inhibitor, NG-monomethyl-L-arginine (L-NMMA) at doses previously shown to inhibit nNOS or both $\mathrm{nNOS}$ and eNOS, respectively. 18 patients already undergoing elective cardiac catheterisation for clinical reasons and found to have normal coronary arteries were included. An intracoronary Doppler flow wire was positioned in the coronary artery for measurement of blood flow velocity whereas 